\title{
Eigenstructure Assignment Method and Its Applications to the Constrained Problem
}

\author{
H. Maarouf, A. Baddou \\ ${ }^{1}$ Mathematics and Computer Sciences Department Sidi Bouzid, Safi, Morocco \\ ${ }^{2}$ Ibn Zohr University, National School of Applied Sciences (ENSA), Agadir, Morocco \\ Email: maarouforama@gmail.com, a.baddou@uiz.ac.ma
}

Received 13 April 2014; revised 17 May 2014; accepted 25 May 2014

Copyright (C) 2014 by authors and Scientific Research Publishing Inc.

This work is licensed under the Creative Commons Attribution International License (CC BY). http://creativecommons.org/licenses/by/4.0/

c) (i) Open Access

\begin{abstract}
A partial eigenstructure assignment method that keeps the open-loop stable eigenvalues and the corresponding eigenspace unchanged is presented. This method generalizes a large class of systems previous methods and can be applied to solve the constrained control problem for linear invariant continuous-time systems. Besides, it can be also applied to make a total eigenstructure assignment. Indeed, the problem of finding a stabilizing regulator matrix gain taking into account the asymmetrical control constraints is transformed to a Sylvester equation resolution. Examples are given to illustrate the obtained results.
\end{abstract}

\section{Keywords}

Sylvester Equation, Linear Continuous-Time Systems, Constrained Control

\section{Introduction}

Eigenstructure assignment method plays a capital role in control theory of linear systems. The state feedback control law is used in this end, leading to change eigenvalues or eigenvectors of the open loop to desired ones in the closed loop. This method is usually realized in order to perform optimal or stabilizing control laws ([1]-[9]). These articles and the references therein constitute a comprehensive summary and an important bibliography on the control of linear systems with input saturation. Indeed, authors have used different concepts leading to many methods for eigenstructure assignment control laws to regulate linear systems with input saturation.

Throughout this paper, we will be interested in continuous time systems of the form

$$
\dot{x}(t)=A x(t)+B u(t) .
$$

The matrices $A$ and $B$ are real and constant: $A \in \mathbb{R}^{n \times n}$ and $B \in \mathbb{R}^{n \times m}$ with $1 \leq m \leq n$. The vector $x(t) \in \mathbb{R}^{n}$ 
represents the state vector of the system and $u(t) \in \mathbb{R}^{m}$ is the control vector. We suppose that the spectrum of the matrix $A$ contains $n-m$ desirable or stable eigenvalues $\lambda_{m+1}, \cdots, \lambda_{n}$ and $m$ undesirable or unstable eigenvalues $\lambda_{1}, \cdots, \lambda_{m}$. We also suppose that the pair $(A, B)$ is stabilizable.

Presence of undesirable eigenvalues makes System (1) unstable and, in [4] or in [10], methods to overcome the instability of System (1), keeping unchanged the open-loop stable eigenvalues and the corresponding eigenspace $\mathbb{F}_{s}$ and replacing the remaining undesirable eigenvalues by other chosen values, were given. But, in these methods, additional conditions on System (1) should be satisfied.

In this paper, we try to get rid of these additional conditions on System (1). First, we should give some outlines on the methods described in [4] or in [10]. In [4], the method, called the inverse procedure, consists of giving a matrix $H \in \mathbb{R}^{m \times m}$ with some desirable or stable spectrum and then computing, when possible, a full rank feedback matrix $F \in \mathbb{R}^{m \times n}$ such that:

$$
F(A+B F)=H F .
$$

and the kernel of $F$ is the stable subspace. In this case, the spectrum of the matrix $A+B F$ is stable since it is constituted by the desirable eigenvalues of $A$ and the chosen spectrum of $H$. In other words, the eigenvalues of $H$ will replace, in the closed-loop, the undesirable eigenvalues of $A$. With the change of variables $z(t)=F x(t)$ in the initial system, we get

$$
\dot{z}(t)=H z(t)
$$

which allows one to focus on the unstable part of this system.

The inverse procedure described in [4] ensures the existence of a matrix $F$ that satisfies the conditions mentioned above and gives a way to compute it under the following conditions:

(a) The matrix $H$ is diagonalizable; that is, there exist linearly independent eigenvectors $\theta_{1}, \cdots, \theta_{m}$ of $H$ associated to some stable eigenvalues $\mu_{1}, \cdots, \mu_{m}$.

(b) The endomorphism induced by the matrix $A$ on the stable subspace is diagonalizable; that is, there exist linearly independent eigenvectors $\xi_{m+1}, \cdots, \xi_{n}$ of $A$ in the subspace $\mathbb{F}_{s}$.

(c) The spectrum of $A$ and the spectrum of $H$ are disjoint.

(d) The matrix

$$
M=\left[\left(\mu_{1} \mathbb{I}_{n}-A\right)^{-1} B \theta_{1}, \cdots,\left(\mu_{m} \mathbb{I}_{n}-A\right)^{-1} B \theta_{m}, \xi_{m+1}, \cdots, \xi_{n}\right]
$$

is invertible.

Under these conditions and according to the method described in [4], the matrix $F$ is unique and is given by

$$
F=\left[\theta_{1}, \cdots, \theta_{m}, 0_{m+1}, \cdots, 0_{n}\right] M^{-1}
$$

where $0_{i}$ is the null vector of $\mathbb{R}^{n}$.

In [10], the condition b) is kept and a new assumption should be fulfilled:

(d'). the matrix $N=\left[B, \xi_{m+1}, \cdots, \xi_{n}\right]$ is invertible.

The method described in [10] consists of computing a matrix $V$ such that its rows span the orthogonal of the stable subspace by taking, for example the first $m$ rows of $N^{-1}$ and then, for a stable matrix $H \in \mathbb{R}^{m \times m}$. Then, according to the method described in [10], the feedback matrix $F$ is given by $F=K V$ where $K$ is the inverse matrix of the solution of the Sylvester equation

$$
\Lambda X-X H=-V B,
$$

and $\Lambda \in \mathbb{R}^{m \times m}$ is a matrix such that $V A=\Lambda V$.

In this paper, we generalize the method described in [10] to a more general class of systems for which the condition d') is not necessary. In fact, no additional condition is needed to deal with System (1). As in [10], the feedback matrix $F$ is always given by $K V$ where $K$ is an invertible matrix of $\mathbb{R}^{m \times m}$ such that $X=K^{-1}$ is the solution of the Sylvester Equation (5).

The methods described in [10] or in [4] are, in fact, partial pole placement methods in which the desirable eigenvalues of the matrix $A$ are kept in the closed-loop. But, it may happen that these desirable eigenvalues are close to the imaginary axis which causes a slow convergence rate to the origin. To overcome this problem, a total pole placement is needed. The technique of augmentation (see [5] and [6]) allows one to perform a total pole placement when the matrix $B$ needs not be of full rank. This is possible with the inverse procedure [4], but not 
with the method described in [10] since, under the condition 1, the matrix $B$ is of full rank.

So, from one hand, the method that we present constitutes a generalization of the two methods described in [10] or in [4] without any other additional condition on the systems and, from another hand, allows one to make, if necessary, a total pole placement by the use of the augmentation technique.

The paper is organized as follows: In Section 1, definitions, notations and some known facts are presented to be used in the sequel. The main results are presented in Section 2 together with an illustrative example. Some particular cases are presented in Section 3. Section 4 is devoted to the total eigenstructure problem with the illustrative example of the double integrator.

\section{Preliminaries}

\subsection{Notations and Definitions}

- $\mathbb{I}_{p}$ is the identity matrix of $\mathbb{R}^{p \times p}$ for $p \in \mathbb{N}^{*}$.

- Matrices of the form $\lambda \mathbb{I}_{p}$ are called scalar matrices for $\lambda \in \mathbb{R}$ and $p \in \mathbb{N}^{*}$.

- If $M$ is a $p \times q$ real matrix, for some $p, q \in \mathbb{N}^{*}$, its transpose, the $q \times p$ real matrix, will be denoted by $M^{\mathrm{T}}$.

- When we view $\mathbb{R}^{n}$ as an euclidean space, the usual inner product $(\cdot \mid \cdot)$ is considered, that is,

$$
(x \mid y)=\sum_{i=1}^{n} x_{i} y_{i},
$$

where $x=\left(\begin{array}{lll}x_{1} & \cdots & x_{n}\end{array}\right)^{\mathrm{T}}$ and $y=\left(\begin{array}{lll}y_{1} & \cdots & y_{n}\end{array}\right)^{\mathrm{T}}$ are two vectors of $\mathbb{R}^{n}$.

- If $S$ is a nonempty subset of $\mathbb{R}^{n}$, its orthogonal will be denoted by $S^{\perp}$, that is,

$$
S^{\perp}=\left\{x \in \mathbb{R}^{n} \mid(x \mid y)=0 \text { for all } y \in S\right\} .
$$

- For a real number $x$, we define

$$
x^{+}=\left\{\begin{array}{ll}
x & \text { if } x \geq 0 \\
0 & \text { otherwise }
\end{array}=\max (x, 0) \text { and } x^{-}=\left\{\begin{array}{ll}
-x & \text { if } x \leq 0 \\
0 & \text { otherwise }
\end{array}=\max (-x, 0) .\right.\right.
$$

- If $M$ is a $p \times q$ real matrix, for some $p, q \in \mathbb{N}^{*}, M_{i, j}$ will denote the coefficient of $M$ corresponding to the $(i, j)$ row-column.

- If $M$ is a $p \times q$ real matrix, for some $p \in \mathbb{N}^{*}, \tilde{M}_{c}$ is the $2 p \times 2 p$ real matrix defined by

$$
\begin{gathered}
\tilde{M}_{c}=\left(\begin{array}{ll}
M_{1} & M_{2} \\
M_{2} & M_{1}
\end{array}\right), \\
\text { where }\left[M_{1}\right]_{i, j}=\left\{\begin{array}{ll}
M_{i, i} & \text { if } i=j, \\
M_{i, j}^{+} & \text {if } i \neq j,
\end{array} \text { and }\left[M_{2}\right]_{i, j}= \begin{cases}0 & \text { if } i=j, \\
M_{i, j}^{-} & \text {if } i \neq j .\end{cases} \right.
\end{gathered}
$$

- For real matrices (or real vectors) $M$ and $N$, we say that $M$ is less than or equal to $N$ if every component of $M$ is less than or equal to the corresponding component of $N$. We then write $M \leq N$.

- If $M$ is a $p \times q$ real matrix, for some $p, q \in \mathbb{N}^{*}$,

(a) ker $M$ is the kernel of $M$ : the subspace of $\mathbb{R}^{q}$ of vectors $X$ such that $M X=0$.

(b) Im $M$ is the image of $M$ : the subspace of $\mathbb{R}^{p}$ spanned by the columns of $M$ and also the set of vectors of the form $M X$ with $X \in \mathbb{R}^{q}$.

- If $M$ is a $p \times p$ real matrix, for some $p \in \mathbb{N}^{*}, \sigma(M)$ denotes its spectrum in the field of complex numbers $\mathbb{C}$.

- If $\lambda$ is a complex number then $\operatorname{Re}(\lambda)$ is the real part of $\lambda$.

\subsection{Some Notes on the Stable-Unstable Subspaces}

1. To System (1), we associate:

(a) The two polynomials

$$
P(x)=\left(x-\lambda_{1}\right) \cdots\left(x-\lambda_{m}\right)
$$




$$
Q(x)=\left(x-\lambda_{m+1}\right) \cdots\left(x-\lambda_{n}\right)
$$

that are factors of the characteristic polynomial of $A$.

In case of $m=n$, polynomial $Q$ is just the constant polynomial 1 .

(b) The two subspaces of $\mathbb{R}^{n} ; \mathbb{F}_{u}=\operatorname{ker} P(A)$ and $\mathbb{F}_{s}=\operatorname{ker} Q(A)$.

In case of $m=n$, the subspace $\mathbb{F}_{s}$ is just the trivial subspace $\operatorname{ker} \mathbb{I}_{n}=\{0\}$.

\section{Remark 1}

1. Since $P$ and $Q$ are coprime, $\mathbb{F}_{u}$ and $\mathbb{F}_{s}$ are complementary subspaces of $\mathbb{R}^{n}$. Moreover, we have

$$
\mathbb{F}_{s}=\operatorname{Im} P(A) \quad \text { and } \quad \mathbb{F}_{u}=\operatorname{Im} Q(A)
$$

and also

$$
\mathbb{F}_{s}^{\perp}=\operatorname{Im} Q\left(A^{\mathrm{T}}\right)=\operatorname{ker} P\left(A^{\mathrm{T}}\right) \quad \text { and } \quad \mathbb{F}_{u}^{\perp}=\operatorname{Im} P\left(A^{\mathrm{T}}\right)=\operatorname{ker} Q\left(A^{\mathrm{T}}\right) .
$$

2. Since pair $(A, B)$ is stabilizable, we have

$$
\mathbb{F}_{u} \subset \sum_{k=0}^{n-1} \operatorname{Im}\left(A^{k} B\right) .
$$

\subsection{Some Notes on Sylvester Equation}

Many problems in analysis and control theory can be solved using the well known of Sylvester equation. This equation is widely studied or used in the literature ([10]-[15]). Since Sylvester equation plays a central role in the development of this work, we shall recall conditions under which it has a unique solution. A Sylvester equation is any equation of the form

$$
M X-X N=C
$$

where $M$ is a $p \times p$ real or complex matrix, $N$ is a $q \times q$ real or complex matrix and $C$ is a $p \times q$ real or complex matrix while matrix $X$ stands for an unknown $p \times q$ real or complex matrix. The following well known result gives a sufficient condition for the existence and uniqueness of a solution of Sylvester Equation (6).

\section{Theorem 1}

If spectrums of matrices $M$ and $N$ are disjoint, $\sigma(M) \cap \sigma(N)=\varnothing$, then Sylvester Equation (6) has a unique solution.

\subsection{System (1) with Constraints on the Control}

Consider System (1) with the assumption that the control $u$ is constrained to be in the region $D$ of $\mathbb{R}^{m}$ defined by

$$
D=\left\{u \in \mathbb{R}^{m} \mid-u_{\min } \leq u \leq u_{\max }\right\}
$$

where $u_{\min }$ and $u_{\max }$ are positive vectors in $\mathbb{R}^{m}$. Note that the region $D$ is a non symmetrical polyhedral set as is generally the case in practical situations. Let us first consider the unconstrained case where the regulator problem for System (1) consists in realizing a feedback law as

$$
u=F x,
$$

where $F$ is chosen in $\mathbb{R}^{m \times n}$ with full rank $m$. In this case, System (1) becomes

$$
\dot{x}=(A+B F) x .
$$

The stability of the closed loop System (8) is obtained if, and only if,

$$
\operatorname{Re}(\lambda)<0
$$

for all eigenvalues $\lambda$ of the matrix $A+B F$. In the constrained case, the approach proposed in ([4]-[6]) consists of giving conditions allowing the choice of a stabilizing controller (7) in such a way that the state is constrained to evolve in a specified region of $\mathbb{R}^{n}$ defined by 


$$
D\left(F, V_{\min }, V_{\max }\right)=\left\{x \in \mathbb{R}^{n} \mid-u_{\min } \leq F x \leq u_{\max }\right\} .
$$

Note that the domain $D\left(F, u_{\min }, u_{\max }\right)$ is bounded only in case of $m=n$. In fact, when $m<n$, the subspace ker $F$ has dimension $n-m>0$ and is a subset of this domain. Suppose now that there is a matrix $H \in \mathbb{R}^{m \times m}$ such that

$$
F(A+B F)=H F
$$

Hence, by letting $z=F x$, we get

$$
\dot{z}=H z
$$

and then $z(t)=\exp (t H) z(0)$. We would get $z(t) \in D$ for all $t \geq 0$ whenever $z(0) \in D$. We say that $D$ is positively invariant with respect to the motion of System (12). More generally, we give the following definition of positive invariance.

\section{Definition 1}

A nonempty subset $\mathscr{P}$ of $\mathbb{R}^{m}$ is said to be positively invariant with respect to the motion of System (12) if, for every initial state $z(0)$ in $\mathscr{P}$, the motion $z(t)$ remains in for every $t \geq 0$.

The following theorem gives necessary and sufficient conditions for domain $D$ to be positively invariant with respect to the motion of System (12).

Theorem 2 ([6])

The domain D is positively invariant with respect to the motion of System (12) if, and only if,

$$
\tilde{H}_{c} U \leq 0
$$

where $U \in \mathbb{R}^{2 m}$ is the real vector $U=\left[\begin{array}{l}u_{\text {max }} \\ u_{\text {min }}\end{array}\right]$.

Till now, we have supposed the existence of a matrix $H$ that satisfies Equation (11). The following result, which does not take into account the constrained problem, gives necessary and sufficient conditions for its existence.

Theorem 3 ([4])

ker $F$ is positively invariant with respect to the motion of System (12) if, and only if, there is a matrix $H \in \mathbb{R}^{m \times m}$ such that Equation (11) is satisfied.

Note that $\operatorname{ker} F$ is positively invariant with respect to the motion of System (12) is the same as $\operatorname{ker} F$ is stable by matrix $A$. If the constrained problem is taken into account, the following theorem gives necessary and sufficient conditions for positive invariance of the domain of states $D\left(F, u_{\min }, u_{\max }\right)$.

Theorem 4 ([6])

The domain $D\left(F, u_{\min }, u_{\max }\right)$ is positively invariant with respect to System (8) if, and only if, there is a matrix $H \in \mathbb{R}^{m \times m}$ such that Equation (11) is satisfied and

$$
\tilde{H}_{c} U \leq 0 .
$$

\section{Main Results}

At first, we compute, and fix in the sequel, some matrix $V \in \mathbb{R}^{m \times n}$ such that its rows span the subspace $\mathbb{F}_{s}^{\perp}$. Since the dimension of the subspace $\mathbb{F}_{s}^{\perp}$ is $m$, the matrix $V$ is of full rank $m$.

Proposition 1 There is a unique matrix $\Lambda \in \mathbb{R}^{m \times m}$ such that $V A=\Lambda V$. This matrix is given by

$$
\Lambda=V A V^{\mathrm{T}}\left(V V^{\mathrm{T}}\right)^{-1}
$$

and its spectrum is $\left\{\lambda_{1}, \cdots, \lambda_{m}\right\}$.

Proof.

Let $f$ be the endomorphism of $\mathbb{R}^{n}$ canonically associated to matrix $A^{\mathrm{T}}$, that is,

$$
\begin{aligned}
f: \mathbb{R}^{n} & \rightarrow \mathbb{R}^{n} \\
x & \mapsto A^{\mathrm{T}} x .
\end{aligned}
$$

The subspace $\mathbb{F}_{s}^{\perp}$ is stable under $f$ (that is, $f(x)$ belongs to $\mathbb{F}_{s}^{\perp}$ whenever $x$ is in $\mathbb{F}_{s}^{\perp}$ ). So, one can define 
the endomorphism $g$ induced by $f$ on $\mathbb{F}_{s}^{\perp}$. Matrix $V^{\mathrm{T}}$, when identified with its column vectors, can be seen as a basis of $\mathbb{F}_{s}^{\perp}$. In this basis, if we denote by $L$ the matrix of $g$, we will have

$$
A^{\mathrm{T}} V^{\mathrm{T}}=V^{\mathrm{T}} L .
$$

Let now $\Lambda=L^{T}$. By transposition of formula (15), we get $V A=\Lambda V$. Since $g$ is induced by $f$ on $\mathbb{F}_{s}^{\perp}=\operatorname{ker} P\left(A^{\mathrm{T}}\right)$, its spectrum is $\left\{\lambda_{1}, \cdots, \lambda_{m}\right\}$ which is also the spectrum of $\Lambda$. Formula (14) derives from the fact that $V$ is of full rank $m$ and shows the uniqueness of the matrix $\Lambda$.

\section{Proposition 2}

For any matrix $F \in \mathbb{R}^{m \times n}$ such that $\operatorname{ker} F=\mathbb{F}_{s}$, there is an invertible matrix $K \in \mathbb{R}^{m \times m}$ such that $F=K V$ and $F$ is of full rank $m$.

\section{Proof.}

Let $F \in \mathbb{R}^{m \times n}$ such that $\operatorname{ker} F=\mathbb{F}_{s}$. Since dimension of $\mathbb{F}_{s}$ is $n-m$, rank formula shows that $F$ is of full rank $m$. Let now $U \in \mathbb{R}^{m}$ such that $F V^{\mathrm{T}} U=0$. Then, $V^{\mathrm{T}} U \in \operatorname{ker} F=\mathbb{F}_{s}$ and then $V V^{\mathrm{T}} U=0$. But matrix $V V^{\mathrm{T}}$ is invertible, so $U=0$ and this shows that matrix $F V^{\mathrm{T}}$ is invertible. Clearly, $K=F V^{\mathrm{T}}\left(V V^{\mathrm{T}}\right)^{-1}$ is invertible. For $x \in \mathbb{R}^{n}$, we have

$$
x=V^{\mathrm{T}}\left(V V^{\mathrm{T}}\right)^{-1} V x+x-V^{\mathrm{T}}\left(V V^{\mathrm{T}}\right)^{-1} V x
$$

Since $V\left(x-V^{\mathrm{T}}\left(V V^{\mathrm{T}}\right)^{-1} V x\right)=0$, we have $x-V^{\mathrm{T}}\left(V V^{\mathrm{T}}\right)^{-1} V x \in \operatorname{ker} V=\operatorname{ker} F \quad$ and then

$$
F x=F V^{\mathrm{T}}\left(V V^{\mathrm{T}}\right)^{-1} V x \text {. So }
$$

$$
K V x=F V^{\mathrm{T}}\left(V V^{\mathrm{T}}\right)^{-1} V V^{\mathrm{T}}\left(V V^{\mathrm{T}}\right)^{-1} V x=F V^{\mathrm{T}}\left(V V^{\mathrm{T}}\right)^{-1} V x=F x .
$$

Since this equality holds for all $x \in \mathbb{R}^{n}$, we have $K V=F$.

Theorem 5 Let $S=\left\{\mu_{1}, \cdots, \mu_{m}\right\}$ be a set of complex numbers stable under complex conjugation such that $S \cap\left\{\lambda_{1}, \cdots, \lambda_{m}\right\}=\varnothing$ and $H \in \mathbb{R}^{m \times m}$ with spectrum $S$. Then, there is matrix $F \in \mathbb{R}^{m \times n}$ of full rank $m$ such that $F(A+B F)=H F$ and $\operatorname{ker} F=\mathbb{F}_{s}$ if, and only if, Sylvester equation

$$
\Lambda X-X H=-V B
$$

has a unique invertible solution $X$ such that $F=X^{-1} V$.

\section{Proof.}

The if part: Let $F \in \mathbb{R}^{m \times n}$ be of full rank matrix such that $F(A+B F)=H F$ and ker $F=\mathbb{F}_{s}$. From Proposition 2, there is an invertible matrix $K \in \mathbb{R}^{m \times m}$ such that $F=K V$. Since $V A=\Lambda V$, we get

$$
\begin{aligned}
\Lambda K^{-1}-K^{-1} H+V B & =K^{-1}(K \Lambda-H K+K V B K) K^{-1} \\
& =K^{-1}(K \Lambda V-H K V+K V B K V) V^{\mathrm{T}}\left(V V^{\mathrm{T}}\right)^{-1} K^{-1} \\
& =K^{-1}(K A-H F+F B F) V^{\mathrm{T}}\left(V V^{\mathrm{T}}\right)^{-1} K^{-1} \\
& =0 .
\end{aligned}
$$

Then $X=K^{-1}$ is an invertible solution to Sylvester Equation (16).

The only if part: Suppose now that Sylvester Equation (16) has an invertible solution $X$ and let $K=X^{-1}$. Matrix $F=K V$ satisfies $\operatorname{ker} F=\operatorname{ker} V=\mathbb{F}_{s}$ because matrix $K$ is invertible and then is of full rank $m$. We also have

$$
\begin{aligned}
F(A+B F)-H F & =K V(A+B K V)-H K V \\
& =K(V A+V B K V)-H K V \\
& =K(\Lambda V+V B K V)-H K V \\
& =K\left(\Lambda K^{-1}+V B-K^{-1} H\right) K V \\
& =0 .
\end{aligned}
$$




\section{Remark 2}

Because we are focusing on the partial assignment problem, eigenvalues of matrix $H$ should be desirable, that is, matrix $H$ should be Hurwitz. The undesirable eigenvalues $\lambda_{1}, \cdots, \lambda_{m}$ of A must all be different from those of H. So, assumption $\left\{\lambda_{1}, \cdots, \lambda_{m}\right\} \cap S=\varnothing$ in Theorem 5 above is necessary.

Example 1 Consider the linear time-invariant multivariable system described by

$$
\dot{x}=A x+B u
$$

with

$$
A=\left(\begin{array}{ccc}
0 & 1 & -7 \\
0 & -1 & 6 \\
4 & 4 & 4
\end{array}\right) \text { and } B=\frac{1}{3}\left(\begin{array}{cc}
-1 & 2 \\
3 & 0 \\
-1 & -1
\end{array}\right) .
$$

The control vector is submitted to the constraint $u \in \Omega$ such that,

$$
\Omega=\left\{u \in \mathbb{R}^{2} /-u_{\min }=\left[\begin{array}{c}
-1.5 \\
-2
\end{array}\right] \leq U \leq\left[\begin{array}{c}
2 \\
2.5
\end{array}\right]=u_{\max }\right\} .
$$

Eigenvalues of $A$ are $\lambda_{1}=\lambda_{2}=2$ and $\lambda_{3}=-1$ and pair $(A, B)$ is stabilizable. We get first the matrix

$$
V=\left(\begin{array}{lll}
3 & 3 & 3 \\
5 & 5 & 4
\end{array}\right)
$$

Note that the rows of $V$ are orthogonal to the eigenvector of $A$ associated to the stable eigenvalue $\lambda_{3}$ of $A$. We, then, choose the matrix

$$
H=\left[\begin{array}{cc}
-\frac{1}{2} & \frac{1}{2} \sqrt{3}-1 \\
\frac{1}{2} \sqrt{3}+1 & -\frac{3}{2}
\end{array}\right] \approx\left[\begin{array}{cc}
-0.50000000 & -0.13397460 \\
1.8660254 & -1.5000000
\end{array}\right] .
$$

This matrix is not diagonalizable and its eigenvalues are $\mu_{1}=\mu_{2}=-1\left(=\lambda_{3}\right)$. Besides, inequality (13) is satisfied. We use the formula $\Lambda=V A V^{\mathrm{T}}\left(V V^{\mathrm{T}}\right)^{-1}$ to get

$$
\Lambda=\left(\begin{array}{ll}
-1 & 3 \\
-3 & 5
\end{array}\right) .
$$

Then, we solve the equation (15) and use the inverse of its solution to get the feedback matrix

$$
\begin{aligned}
F & =X^{-1} V=\left[\begin{array}{ccc}
1 / 6+\frac{5}{18} \sqrt{3} & 1 / 6+\frac{5}{18} \sqrt{3} & 1 / 3+2 / 9 \sqrt{3} \\
-\frac{11}{6}-\frac{5}{18} \sqrt{3} & -\frac{11}{6}-\frac{5}{18} \sqrt{3} & -5 / 3-2 / 9 \sqrt{3}
\end{array}\right] \\
& \approx\left[\begin{array}{lll}
0.64779190 & 0.64779190 & 0.71823350 \\
-2.3144585 & -2.3144585 & -2.0515669
\end{array}\right] .
\end{aligned}
$$

Finally, -1 is the unique eigenvalue of the matrix $A+B F$.

Figure 1 shows that, starting from two different and admissible controls, the corresponding trajectories, in the control space, converge to the origin without saturations.

\section{Particular Cases}

\subsection{Case of Single Input Linear Systems}

We discuss the particular case of single input linear systems, that is, when $m=1$. As described in the general case, we start by computing matrix $V$ which is in case of $m=1$ a row vector that is orthogonal to $\mathbb{F}_{s}$ and is easy to get. Matrix $\Lambda$ is only the real $\lambda_{1} \geq 0$; the unique undesirable eigenvalue of $A$. If we choose $H$ to be a 


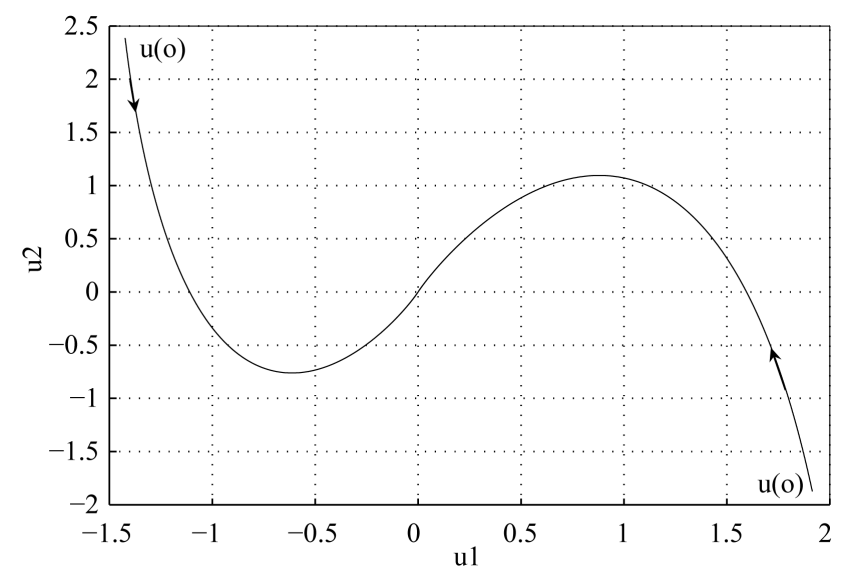

Figure 1. Trajectories of the system $\dot{u}(t)=H u(t)$ from two different initial controls in $\Omega$.

negative real number, Theorem 5 ensures that all matrices $F$ (row vectors) are of the form $K V$ where $K^{-1}$ is a nonzero real solution to the simple "Sylvester equation"

$$
\lambda_{1} x-x H=-V B=x\left(\lambda_{1}-H\right) .
$$

This equation has a nonzero solution if, and only if, the real number $V B$ is nonzero. That is,

$$
x=\frac{V B}{H-\lambda_{1}} .
$$

Formula $V A=\lambda_{1} V$ shows that $V$ is a left eigenvector of $A$ associated to the undesirable eigenvalue $\lambda_{1}$ and then $V A^{k} B=\lambda_{1}^{k} V B$ for every $k \in \mathbb{N}$. But pair $(A, B)$ is stabilizable, so

$$
\mathbb{F}_{u}=\operatorname{ker}\left(A-\lambda_{1} \mathbb{I}_{n}\right) \subset \sum_{k=1}^{n-1} \operatorname{Im}\left(A^{k} B\right) .
$$

If we suppose that $V B=0$, then we should have

$$
V \mathbb{F}_{u} \subset \sum_{k=1}^{n-1} \operatorname{Im}\left(\lambda_{1}^{k} V B\right)=\{0\}
$$

and then $V \mathbb{F}_{u}=\{0\}$. We also have $V \mathbb{F}_{s}=\{0\}$ and $\mathbb{R}^{n}=\mathbb{F}_{u} \oplus \mathbb{F}_{s}$. This shows that $V=0$ which is not true. As a consequence, we have the following result.

Proposition 3 For a given left eigenvector $V$ of $A$ associated to the unique eigenvalue $\lambda_{1}$ and for a negative real number $h$, we have $V B \neq 0$ and matrix $F$ given by

$$
F=\frac{h-\lambda_{1}}{V B} V .
$$

The spectrum of $A+B F$ is then $\left\{h, \lambda_{2}, \cdots, \lambda_{n}\right\}$.

\subsection{Case Where the Matrix VB Is Nonsingular}

We have seen in the last paragraph that, when $m=1$, a necessary and sufficient condition for Sylvester Equation (15) to have nonsingular solution (nonzero real number in fact) is that the real $V B$ is nonzero. We also have seen that this last condition; $V B \neq 0$, is equivalent to the fact that pair $(A, B)$ is stabilizable. In case of $m \geq 2$, it may happen that pair $(A, B)$ is stabilizable but matrix $V B$ is singular as will show the following example.

\section{Example 2}


Consider System (1) with

$$
A=\left[\begin{array}{ccc}
4 & 0 & -1 \\
4 & -1 & -5 \\
4 & 0 & 0
\end{array}\right] \text { and } B=\left[\begin{array}{cc}
1 & 1 \\
-4 & -1 \\
-1 & -1
\end{array}\right] .
$$

Eigenvalues of $A$ are $\lambda_{1}=\lambda_{2}=2$ and $\lambda_{3}=-1$. The last desirable eigenvalue $\lambda_{3}$ is associated to the eigenvector $\left[\begin{array}{lll}0 & 1 & 0\end{array}\right]^{\mathrm{T}}$ of $A$ that spans the subspace $\mathbb{F}_{s}$. Matrix $V$ is then

$$
V=\left[\begin{array}{lll}
1 & 0 & 1 \\
1 & 0 & 2
\end{array}\right]
$$

Matrix $V B$ is singular since it is

$$
V B=\left[\begin{array}{cc}
0 & 0 \\
-1 & -1
\end{array}\right]
$$

Controllability matrix is given by

$$
\left[B, A B, A^{2} B\right]=\left[\begin{array}{cccccc}
1 & 1 & 5 & 5 & 16 & 16 \\
-4 & -1 & 13 & 10 & -13 & -10 \\
-1 & -1 & 4 & 4 & 20 & 20
\end{array}\right]
$$

and is of full rank 3 . This shows that pair $(A, B)$ is stabilizable since it is even controllable.

The case of matrix $V B$ is nonsingular can be seen as a general case of $V B \neq 0$ when $m=1$. So, it deserves a special study that will be the aim in the sequel of this paragraph. In the following theorem, we give a necessary and sufficient condition for matrix $V B$ to be nonsingular.

\section{Theorem 6}

Matrix $V B$ is nonsingular if, and only if, $\operatorname{Im}(B)$ and $\mathbb{F}_{s}$ are complementary subspaces of $\mathbb{R}^{n}$; that is, $\mathbb{R}^{n}=\operatorname{Im}(B) \oplus \mathbb{F}_{s}$.

\section{Proof.}

The if part: Since $V B$ is nonsingular, matrix $B$ is of full rank $m$. To complete the proof of the if part, we shall show that $\{0\}=\operatorname{Im}(B) \cap \mathbb{F}_{s}$ since the dimension of the subspace $\mathbb{F}_{s}$ is $n-m$. Recall that $V X=0$ for any vector $X$ in $\mathbb{F}_{s}$. If now $X$ is both in $\mathbb{F}_{s}$ and $\operatorname{Im}(B)$, then there is $U \in \mathbb{R}^{m}$ such that $X=B U$ and then

$$
0=V X=V B U .
$$

This shows that $U=0$ since $V B$ is nonsingular and, then, $X=0$.

The only if part: From the fact that $V X=0$ for any vector $X$ in $\mathbb{F}_{s}$; that is $V \mathbb{F}_{s}=\{0\}, V$ is of full rank $m$; that is $V \mathbb{R}^{n}=\mathbb{R}^{m}$. Moreover, from the fact that $\mathbb{R}^{n}=\operatorname{Im}(B) \oplus \mathbb{F}_{s}$, we get

$$
\mathbb{R}^{m}=V \mathbb{R}^{n}=V \operatorname{Im}(B)+V \mathbb{F}_{s}=\operatorname{Im}(V B)
$$

since $V$ is linear. This shows that the rank of $V B$ is $m$ and that it is nonsingular.

The following theorem gives another method to get a partial pole assignment under the assumption that $V B$ is nonsingular.

Theorem 7

Suppose that matrix $V B$ is nonsingular and let matrix $L \in \mathbb{R}^{m \times m}$ be such that $\sigma(L)=\left\{\mu_{1}, \cdots, \mu_{m}\right\}, \sigma(L) \cap\left\{\lambda_{1}, \cdots, \lambda_{m}\right\}=\varnothing$ and $L-\Lambda$ is nonsingular. Then,

$$
\sigma(A+B F)=\left\{\mu_{1}, \cdots, \mu_{m}, \lambda_{m+1}, \cdots, \lambda_{n}\right\}
$$

where $F=(V B)^{-1}(L-\Lambda) V$.

Proof.

Let $K=(V B)^{-1}(L-\Lambda)$ and $H=K L K^{-1}$. Then,

$$
\begin{aligned}
\Lambda K^{-1}-K^{-1} H & =\left(\Lambda-K^{-1} H K\right) K^{-1} \\
& =(\Lambda-L)(L-\Lambda)^{-1} V B \\
& =-V B .
\end{aligned}
$$


This shows that $K^{-1}=(L-\Lambda)^{-1} V B$ is a nonsingular solution to Sylvester Equation (16) and, by Theorem 5, that matrix $F=(V B)^{-1}(L-\Lambda) V$ satisfies

$$
\sigma(A+B F)=\left\{\mu_{1}, \cdots, \mu_{m}, \lambda_{m+1}, \cdots, \lambda_{n}\right\}
$$

\section{Example 3}

Consider System (1) with

$$
A=\left[\begin{array}{ccc}
-4 & -9 & -9 \\
3 & 14 & 15 \\
1 & -6 & -7
\end{array}\right] \text { and } B=\left[\begin{array}{cc}
3 & 2 \\
0 & -2 \\
-1 & 1
\end{array}\right] \text {. }
$$

Eigenvalues of $A$ are $\lambda_{1}=\lambda_{2}=2$ and $\lambda_{3}=-1$. The last desirable eigenvalue $\lambda_{3}$ is associated to the eigenvector $\left[\begin{array}{lll}0 & -1 & 1\end{array}\right]^{\mathrm{T}}$ of $A$ that spans the subspace $\mathbb{F}_{s}$. Matrix $V$ is then

$$
V=\left[\begin{array}{lll}
1 & 1 & 1 \\
0 & 1 & 1
\end{array}\right] .
$$

Matrix $V B=\left[\begin{array}{cc}2 & 1 \\ -1 & -1\end{array}\right]$ is nonsingular and matrix $\Lambda$ is given by

$$
\Lambda=V A V^{\mathrm{T}}\left(V V^{\mathrm{T}}\right)^{-1}=\left[\begin{array}{cc}
0 & -1 \\
4 & 4
\end{array}\right]
$$

Now choose $\mu_{1}=\mu_{2}=-2$ that are the eigenvalues of the matrix $H=\left[\begin{array}{cc}-6 & -4 \\ 4 & 2\end{array}\right]$. Then,

$$
F=(V B)^{-1}\left(-2 \mathbb{I}_{2}-\Lambda\right) V=\left[\begin{array}{ccc}
-6 & -11 & -11 \\
10 & 21 & 21
\end{array}\right] \text {. }
$$

\section{A Total Eigenstructure Problem}

Note that, when $m$ and $n$ are equal, matrix $V$ described in the last section is now any invertible matrix of $\mathbb{R}^{n \times n}$. That is why, we suppose $V=\mathbb{I}_{n}$. Then matrix $\Lambda$ is simply $A$ and Sylvester Equation (15) becomes

$$
A X-X H=-B
$$

where $H$ is a real $n \times n$ matrix with desirable spectrum. So, if the unique solution $X$ of Equation (20) is invertible, then the spectrum of $A+B F$ and the one of $H$ are equal, where matrix $F$ is $X^{-1}$. Suppose now that $m<n$. The technique of augmentation (see [5] and [6]) consists of augmenting the matrix $B$ by adding zeros in order to get a new matrix $B_{1} \in \mathbb{R}^{n \times n}$. One should also complete the control vector $u$ by fictive real numbers to get $u_{1} \in \mathbb{R}^{n}$. We also replace the assumption: "the pair $(A, B)$ is stabilizable" by "the pair $(A, B)$ is controllable.” System (1) which becomes

$$
\dot{x}=A x+B_{1} u_{1}=A x+B u
$$

does not change. The matrix $H$ is now a real $n \times n$ matrix with some desirable spectrum $S=\left\{\mu_{1}, \cdots, \mu_{n}\right\}$ which does not contain any eigenvalue of matrix $A$. If the unique solution $X$ of Sylvester equation

$$
A X-X H=-B_{1}
$$

is invertible, we let $F=X^{-1}$. The matrices $A+B_{1} F$ and $H$ have the same spectrum $S$. If $F_{1}$ is the matrix of the first $m$ rows of $F$, then $A+B_{1} F=A+B F_{1}$ assigns the spectrum of $A$ to $S$.

Example 4

Consider the problem of stabilization of the following double integrator system

$$
\dot{x}(t)=A x(t)+B u(t)
$$

with 


$$
A=\left[\begin{array}{ll}
0 & 1 \\
0 & 0
\end{array}\right] \text { and } B=\left[\begin{array}{l}
0 \\
1
\end{array}\right]
$$

subject to the constraint $-2 \leq u(t) \leq 1.5$. The matrix $B$ and the vector $u(t)$ are respectively augmented to

$$
B_{1}=\left[\begin{array}{ll}
1 & 0 \\
0 & 0
\end{array}\right] \text { and } u_{1}(t)=\left[\begin{array}{c}
u(t) \\
z(t)
\end{array}\right]
$$

such that $-z_{1} \leq z(t) \leq z_{2}$ where $z_{1}$ and $z_{2}$ are fictive positive real numbers. Let, for example, $z_{1}=2$ and $z_{2}=2.5$ to get the domain $\Omega$ in the Example 1 . We choose the matrix

$$
H=\frac{1}{2}\left[\begin{array}{cc}
-3+\sqrt{2} & \sqrt{2}-2 \\
\sqrt{2}+2 & -3-\sqrt{2}
\end{array}\right] \approx\left[\begin{array}{cc}
-0.79289320 & -0.29289320 \\
1.7071068 & -2.2071068
\end{array}\right]
$$

for which the eigenvalues are $\mu_{1}=\mu_{2}=-1.5$ and the inequality (13) is satisfied. Then, we solve the Sylvester equation (20) and get

$$
F=X^{-1}=-\frac{3}{4}\left[\begin{array}{cc}
3 & 4 \\
12+\frac{15}{2} \sqrt{2} & 10+8 \sqrt{2}
\end{array}\right] .
$$

The feedback matrix $F_{1}$, which represents the first row of $F$, is then

$$
F_{1}=-\frac{3}{4}\left[\begin{array}{ll}
3 & 4
\end{array}\right] .
$$

The spectrum of $A+B F_{1}$ is then $\{-1.5,-1.5\}$.

As in Figure 1 but in the state space, Figure 2 plots two trajectories in the state space starting from two different and admissible initial states and, thanks to the asymptotic stability of the system and the invariance positive property, shows the state convergence to the origin without leaving the domain imposed by the constraints.

\section{Conclusion}

A method for partial or total eigenstructure assignment problem was presented and examples to illustrate the method were given. The method uses Sylvester equation to find the feedback matrix $F$ when some matrix $H$ is given with a desirable spectrum that will replace all the undesirable eigenvalues of the initial matrix $A$ of System (1) in the closed-loop. This method generalizes the one proposed in [10] without additional conditions on Sys-

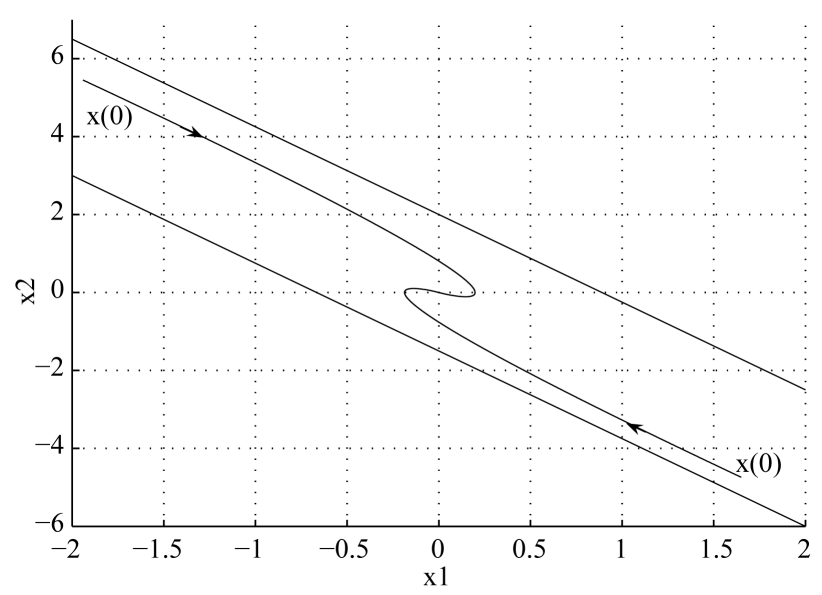

Figure 2. Trajectories of the system $\dot{x}(t)=\left(A+B F_{1}\right) x(t)$ from two different initial states $x(0)$ such that $F_{1} x(0) \in \Omega$. 
tem (5) and allows us to deal with the problem of asymmetrical constraints on the control vector. Examples to show its importance are presented.

\section{References}

[1] Ait Rami, M., El Faiz, S., Benzaouia, A. and Tadeo, F. (2009) Robust Exact Pole Placement via an LMI-Based Algorithm. IEEE Transactions on Automatic Control, 54, 394-398. http://dx.doi.org/10.1109/TAC.2008.2008358

[2] Bachelier, O., Bosche, J. and Mehdi, D. (2006) On Pole Placement via Eigenstructure Assignment Approach. IEEE Transactions on Automatic Control, 51, 1554-1558. http://dx.doi.org/10.1109/TAC.2006.880809

[3] Sussmann, H.J., Sontag, E. and Yang, Y. (1994) A General Result on Stabilization of Linear Systems Using Bounded Control. IEEE Transactions on Automatic Control, 39, 2411-2424. http://dx.doi.org/10.1109/9.362853

[4] Benzaouia, A. (1994) The Resolution of the Equation $X A+X B X=H X$ and the Pole Assignment Problem. IEEE Transactions on Automatic Control, 40, 2091-2095. http://dx.doi.org/10.1109/9.328817

[5] Benzaouia, A. and Burgat, C. (1988) Regulator Problem for Linear Discrete-Time Systems with Nonsymmetrical Constrained Control. International Journal of Control, 48, 2441-2451. http://dx.doi.org/10.1080/00207178808906339

[6] Benzaouia, A. and Hmamed, A. (1993) Regulator Problem for Linear Continuous-Time Systems with Nonsymmetrical Constrained Control. IEEE Transactions on Automatic Control, 38, 1556-1560.

http://dx.doi.org/10.1109/9.241576

[7] Blanchini, F. (1999) Set Invariance in Control. Automatica, 35, 1747-1767. http://dx.doi.org/10.1016/S0005-1098(99)00113-2

[8] Gutman, P.O. and Hagander, P. (1985) A New Design of Constrained Controllers for Linear Systems. IEEE Transactions on Automatic Control, 30.

[9] Lu, J., Chiang, H. and Thorp, J.S. (1991) Partial Eigenstructure Assignment and Its Application to Large Scale Systems. IEEE Transactions on Automatic Control, 36.

[10] Baddou, A., Maarouf, H. and Benzaouia, A. (2013) Partial Eigenstructure Assignment Problem and Its Application to the Constrained Linear Problem. International Journal of Systems Science, 44, 908-915. http://dx.doi.org/10.1080/00207721.2011.649364

[11] Maarouf, H. and Baddou, A. (2012) An Eigenstructure Assignment Method, Publication and Oral Presentation at the International Symposium on Security and Safety of Complex Systems, Agadir.

[12] Barlaw, J.B., Manahem, M.M. and O’Leary, D.P. (1992) Constrained Matrix Sylvester Equations. SIAM Journal on Matrix Analysis and Applications, 13, 1-9. http://dx.doi.org/10.1137/0613002

[13] Ding, F. and Chen, T. (2005) Iterative Least-Squares Solutions of Coupled Sylvester Matrix Equations. Systems and Control Letters, 54, 95-107. http://dx.doi.org/10.1016/j.sysconle.2004.06.008

[14] Gardiner, J.D., Laub, A.J., Amato, J.J. and Moler, C.B. (1992) Solution to the Sylvester Matrix Equation AXB ${ }^{T}+$ $\mathrm{CXD}^{\mathrm{T}}=$ E. ACM Transactions on Mathematical Software, 18, 223-231. http://dx.doi.org/10.1145/146847.146929

[15] Teran, F.D. and Dopico, F.M. (2011) Consistency and Efficient Solution of the Sylvester Equation for Congruence. International Linear Algebra Society, 22, 849-863. 